\title{
A LysM effector protein from the basidiomycete Rhizoctonia solani contributes to virulence through suppression of chitin-triggered immunity
}

\author{
Fredrik Dölfors $^{1} \cdot$ Louise Holmquist $^{1,2} \cdot$ Christina Dixelius $^{1} \cdot$ Georgios Tzelepis $^{1}$
}

Received: 27 February 2019 / Accepted: 29 April 2019 / Published online: 10 May 2019

(c) The Author(s) 2019

\begin{abstract}
Rhizoctonia solani is a fungal species that belongs to the fungal division Basidiomycota. It is a soil-borne pathogen that attacks a broad range of plant species and crops. Disease symptoms are commonly seen as damping off of seedlings and root rot, although it can infect plants at any developmental stage. Despite the severity of this disease, many aspects in $R$. solani infection biology remain unclear. Here we investigated the role of a LysM effector, previously predicted from the genome of a $R$. solani AG2-2IIIB strain that has sugar beet as a host. Gene expression analysis showed that RsLysM was highly induced upon sugar beet infection. When RsLysM was heterologously expressed in Cercospora beticola, necrotic lesion size and fungal colonization ability were increased, indicating a role in virulence. RsLysM displayed chitin-binding affinity and suppression of chitin-triggered immunity but could not protect hyphae from hydrolysis. Overall, this study is the first characterization of a LysM effector from Basidiomycota, suggesting that this necrotrophic fungal species relies on perturbation of chitin-triggered immunity to establish a successful infection.
\end{abstract}

Keywords Cercospora beticola $\cdot$ Effectors $\cdot$ Heterologous expression $\cdot$ LysM $\cdot$ Sugar beet

\section{Introduction}

Rhizoctonia species (in Greek "root-killer") are important soil-borne pathogens causing substantial yield losses in a wide range of crops including cereals, soybean, potato and sugar beet (Anderson 1982; Adams 1988). R. solani incites crown and root rot in sugar beet, leaf spot and root rot in tobacco, sheath blight in rice and black scurf in potato (Ceresini et al. 2002; Bernardes-de-Assis et al. 2009; Buhre et al.

Communicated by S. Hohmann.

Electronic supplementary material The online version of this article (https://doi.org/10.1007/s00438-019-01573-9) contains supplementary material, which is available to authorized users.

Georgios Tzelepis

Georgios.Tzelepis@slu.se

1 Department of Plant Biology, Uppsala BioCenter, Linnean Center for Plant Biology, Swedish University of Agricultural Sciences, P.O. Box 7080, 75007 Uppsala, Sweden

2 MariboHilleshög Research AB, Säbyholmsvägen 24, 26191 Landskrona, Sweden
2009; Gonzalez et al. 2011). The fungus produces persistent sclerotia, which are the major infestation source together with fungal-infested plant debris in the soil. Rhizoctonia solani commonly infects roots and hypocotyls, but all types of plant organs can be colonized by mycelia. The sexual stage (Thanatephorus cucumeris) is extremely rare (Adams and Butler 1983) and conidia formation is lacking. This pathogen is classified into different anastomosis groups (AGs) based on hyphal cell wall fusion between different isolates. Some AGs are further divided into subgroups based on host range, colony morphology, pathogenicity, zymogram patterns and other characteristics (Ogoshi 1987). Despite the economic impact of $R$. solani, little is known about mechanisms behind fungal colonization and growth on host tissue. A general view is that $R$. solani hyphae adhere to the host plant surface and enter the outer cell layers by the formation of penetration cushions (Gonzalez et al. 2011), a process followed by the growth of invasive hyphae that ramify through the host tissue. Genomic data are presently available from five different $R$. solani strains with cereal and dicot plant hosts (Zheng et al. 2013; Cubeta et al. 2014; Hane et al. 2014; Wibberg et al. 2013, 2016a, b), but hypotheses on 
gene functions are difficult to test since $R$. solani is not easily amenable to molecular manipulations.

Effectors are small-secreted proteins deployed by pathogens to manipulate plant defense barriers to establish and promote further growth on the selected host (Stergiopoulos and de Wit 2009; Vleeshouwers and Oliver 2014; Lo Presti et al. 2015). Secreted proteins promoting disease were first observed in human pathogenic bacteria (Preston 2007). As more genomes of plant pathogens have been sequenced, the prediction of secreted proteins has increased substantially. A plethora of strategies are employed; effectors suppress and block early plant defense responses such as the hypersensitive response (HR) (Hemetsberger et al. 2012), mask hyphae from recognition or protect them from plant chitinases (van den Burg et al. 2006; de Jonge and Thomma 2009) or promote necrosis (Qutob et al. 2006). Key molecules acting in various pathways can also be hijacked impacting gene activation and hormone levels (Weiberg et al. 2013; Ma and Ma 2016). Many aspects on effector function during plant-microbe interactions remain unclear and host targets are most likely more than one.

Sugar beet is mainly attacked by AG2-2IIIB strains. The disease can appear in different forms and with different symptoms, root and crown rot, damping off or as foliar blight (Bolton et al. 2010). The demand for high-yielding sugar beet cultivars with resistance to $R$. solani is increasing. Effects of a milder and more humid climate are seen in temperate regions influencing the length of crop seasons and simultaneously allowing pathogens to multiply for longer periods. Moreover, the extent of $R$. solani AG2-2IIIB soil inoculum is expected to increase in regions particularly where sugar beet and maize are overlapping in the crop rotation schemes, since maize can act as a host and thus propagate the pathogen (Buddemeyer et al. 2004; Schulze et al. 2016). The $R$. solani AG2-2IIIB strain has a predicted genome size of $56.02 \mathrm{Mb}$ and 11,897 protein-encoding genes (Wibberg et al. 2016a, b). Here we refined the secretome dataset and identified only one putative lysin motif (LysM) effector in the genome. This candidate effector (RsLysM) has been chosen, since the role of LysM effectors in plant pathogenic basidiomycetes is unknown. They have been previously characterized in ascomycetes with a hemibiotrophic lifestyle, thus we hypothesized that further characterization of RsLysM would generate new insights into the $R$. solani infection process. The RsLys $M$ gene was highly induced in infected sugar beet seedlings and heterologous expression of this gene in the sugar beet pathogen Cercospora beticola led to a significant increase of fungal biomass in infected plant tissues. We further showed that this effector is a chitin-binding protein, able to suppress burst of reactive oxygen species (ROS) induced by chitin, further supporting the concept of a universal role of LysM effectors in perturbation of chitintriggered immunity.

\section{Materials and methods}

\section{Fungal isolates and sequence analysis}

Rhizoctonia solani AG2-2IIIB isolate BBA 69670 (DSM 101808) (Wibberg et al. 2016a) was used in this study. Rhizoctonia solani inoculum for soil infestation was prepared on media containing perlite, corn flour, and water (1:1:5). Cercospora beticola isolate Ty1 was grown on potato dextrose agar plates at $22{ }^{\circ} \mathrm{C}$ in darkness and sporulation was induced on tomato growth extract medium (Marcuzzo et al. 2015). Presence of conserved domains was searched using the SMART 6.0 tool (Letunic et al. 2009), followed by SignalP 4.1 for signal peptide prediction (Petersen et al. 2011).

\section{Quantitative reverse transcriptase-polymerase chain reaction (qRT-PCR)}

Three-week-old sugar beet plants were transplanted in soil infested with $R$. solani mycelia (10:1 ratio of fresh soil: inoculum). Total RNA was extracted from the plants at 4,5 , 6 and 7 days post-inoculation (dpi) using the RNeasy Plant Mini Kit (Qiagen) according to manufacturer's instructions, while $R$. solani mycelia grown on potato dextrose broth were used as fungal control. qRT-PCR was run as described previously (Tzelepis et al. 2012) and primers are listed in Table 1. Expression was normalized to $R$. solani $G 3 P D H$ transcript levels (Chamoun et al. 2015) and relative expression values were calculated according to the $2^{-\Delta \Delta C_{t}}$ method (Livak and Schmittgen 2001).

\section{Construction of Cercospora beticola transgenic strains and virulence assays}

Vectors were designed using the In-Fusion HD cloning kit (Takara Bio). The RsLysM sequence was PCR amplified from $R$. solani cDNA using high fidelity Phusion Taq polymerase (Thermo Scientific). The destination vector was the pRFHUE-eGFP, conferring resistance to hygromycin (Crespo-Sempere et al. 2011). The RsLysM sequence was tagged with GFP at C-terminal and transformed to $C$. beticola using an Agrobacterium-mediated protocol (Utermark and Karlovsky 2008). Primers are listed in Table 1. Three independent strains expressing the RsLysM gene were selected for further analysis. Western blot analysis was also conducted on positive $C$. beticola transgenic strains to confirm the protein expression. Strains were grown on potato dextrose broth (PDB, Difco) for 7 days and proteins were extracted from mycelia using the GTEN extraction buffer (25 mM Tris pH 7.5, 1 mM EDTA, $150 \mathrm{mM} \mathrm{NaCl}, 2 \% \mathrm{w} / \mathrm{v}$ 
Table 1 Primer sequences used in the current study

\begin{tabular}{|c|c|c|c|c|}
\hline Primer name & Forward primer $5^{\prime}-3^{\prime}$ & Reverse primer $5^{\prime}-3^{\prime}$ & $\begin{array}{l}\text { Annealing } T_{\mathrm{m}} \\
\left({ }^{\circ} \mathrm{C}\right)\end{array}$ & References \\
\hline \multicolumn{5}{|l|}{ RT-qPCR } \\
\hline RsLysM & gtcetccegcagctacgecttac & cgggttgcttgttctcaggacttg & 58 & Wibberg et al. (2016b) \\
\hline$G 3 P D H$ & accgttatgggettgtctttcctt & cccgettggetggaatagtaacg & 58 & Chamoun et al. (2015) \\
\hline \multicolumn{5}{|c|}{ DNA quantification } \\
\hline Bv elf-1 & cacgggaaatcaacgctcac & ttcatcggcacgggtatcag & 58 & This study \\
\hline Cbactin & acatggctggtcgtgaatttg & tgtccgtcaggaagctcgta & 58 & Nikou et al. (2009) \\
\hline \multicolumn{5}{|c|}{ Overexpression strains } \\
\hline RsLysM+ & $\begin{array}{l}\text { gcagacatcacccgggatgtttac- } \\
\text { taaactactcattgcttcggc }\end{array}$ & agtcctcagccccggggtagccgtcatcgttgggg & 58 & This study \\
\hline \multicolumn{5}{|c|}{ Protein expression } \\
\hline RsLysM & $\begin{array}{l}\text { cggtatgaattcatgcatcatcatcat- } \\
\text { catcataccgggtgtgctcgaac- } \\
\text { ctatac }\end{array}$ & cggtatgcggecgettattattagtagccgtcatcgttgggg & 55 & This study \\
\hline
\end{tabular}

PVPP, 10 mM DTT, 0.1\% Tween-20 and protease inhibitors (Roche). Proteins from culture filtrates were concentrated using ultrafiltration centrifugal tubes (ThermoFisher) at $5000 \mathrm{~g}$. Detection of GFP was carried out using the monoclonal anti-GFP HRP antibody (Santa Cruz Biotechnology).

For virulence assays, leaves of 3-week-old sugar beet plants were inoculated with $10^{5}$ C. beticola conidia/ml $\mathrm{H}_{2} \mathrm{O}$ from transgenic and wild-type (Ty1) strains. The conidia solution contained $0.02 \%$ Tween-20 to increase adherence. Two leaves from each plant were infected, and four plants were included in each biological replicate. In total, three biological replicates were used from three independent strains $(R s L y s M+)$. Infected plants were grown under high humidity conditions and lesion area was monitored at 7 dpi, and quantified using the ImageJ software version $1.51 \mathrm{n}$ (National Institute of Health, USA). Total genomic DNA was extracted from infected leaves using a CTAB-mediated protocol (Möller et al. 1992). Fungal DNA was quantified using the $C$. beticola actin (act) reference gene and normalized with $B$. vulgaris elongation factor (elf-l) gene. Primers are listed in Table 1.

\section{Expression of RsLysM in Pichia pastoris}

The RsLysM and Avr4 genes from R. solani and Cladosporium fulvum, respectively, were cloned in the pPic9 expression vector with the N-terminal His tag and transformed into the Pichia pastoris strain GS115. Primers are listed in Table 1. A positive clone was cultured in a Bioflo 300 fermenter under growth conditions previously described by Rooney et al. (2005). His-tagged protein was purified using a Ni-NTA column (Qiagen), and concentration was determined using the Pierce BCA Protein assay kit (Thermo Fisher Scientific).

\section{Affinity precipitation, reactive oxygen species and hyphal protection assay}

A chitin-binding assay of the RsLysM effector protein was performed as earlier described (de Jonge et al. 2010). Pure protein $(20 \mu \mathrm{g} / \mathrm{ml})$ was incubated with $3 \mathrm{mg}$ insoluble polysaccharides (crab shell chitin, chitosan, xylan or cellulose) in a total volume of $800 \mu \mathrm{l}$ water. After $3 \mathrm{~h}$ of incubation, the mixture was centrifuged at $13,000 \mathrm{~g}$. The pellet (bound fraction) and supernatant (non-bound fraction) were analyzed on a SDS polyacrylamide gel. Western blot analysis was run to confirm the presence of RsLysM protein in the two fractions using the anti-His HRP monoclonal antibody (Thermo Fisher). For suppression of chitin-induced oxidative burst of reactive oxygen species (ROS), the protocol by de Jonge et al. (2010) was used. Here leaf disks, from Nicotiana benthamiana plants, were inoculated with assay buffer [68 $\mu \mathrm{l} / \mathrm{ml}$ luminol (Sigma) and $40 \mu \mathrm{l} / \mathrm{ml}$ horseradish peroxidase (Sigma)] with/without chitin oligomers (GlcNAc) 6 and the suppression of ROS was analyzed using $10 \mu \mathrm{M}$ RsLysM protein. For hyphal protection, a protocol described by Kohler et al. (2016) was used. $10 \mu \mathrm{M}$ and $20 \mu \mathrm{M}$ of RsLysM protein were incubated with germinated conidia from Trichoderma virens, followed by addition of 0.1 $\mathrm{U}$ bacterial chitinase (Sigma) and $10 \mathrm{U}$ zymolyase (Zymo Research). The Avr4 effector protein $(5 \mu \mathrm{M})$ and BSA were used as positive and negative controls, respectively. Images were taken $6 \mathrm{~h}$ post-inoculation (hpi). 


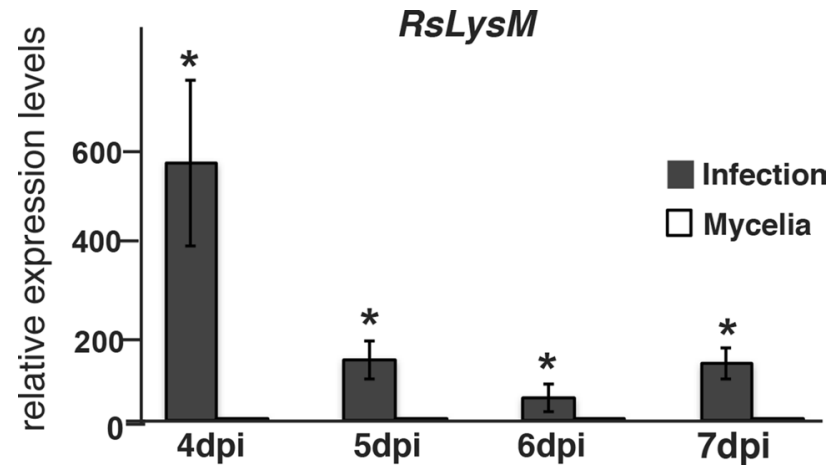

Fig. 1 Transcription analysis of the RsLysM effector. Relative transcript levels of the $R$. solani AG2-2IIIB RsLysM gene during sugar beet infection. Sugar beet plants were grown in infested soil for 4, 5, 6 and 7 days before harvest for qRT-PCR. cDNA from $R$. solani mycelia grown in PDB medium was used as comparison. The G3PDH gene was used as internal standard. Error bars represent SD based on at least three biological replicates. Asterisks (*) indicate statistically significant differences between columns at the same time point according to Fisher's test $(p$ value $<0.01)$

\section{Results}

\section{The RsLysM is highly induced upon sugar beet infection}

In the previous genome analysis of a $R$. solani AG2-2IIIB isolate, 126 predicted secreted and cysteine-rich proteins were identified (Wibberg et al. 2016b). To further reduce the list of effector candidates, only small proteins $(<400$ amino acids) were kept, resulting in 61 predicted sequences. Only one gene (RSOLAG22IIIB_4067), named RsLysM, encoding for a putative LysM effector was identified. Analysis of sugar beet plants, grown in $R$. solani $(R s)$ infested soil, revealed high induction of the RsLysM gene already at 4 dpi suggesting an important role in $R$. solani virulence (Fig. 1).

\section{The RsLysM promotes virulence upon heterologous expression in Cercospora beticola}

The amino acid sequence of RsLysM was analyzed for conserved domains. The SMART tool predicted two CBM50 modules (IPR002482; LysM peptidoglycan binding) in the RsLysM effector. A signal peptide was also predicted in N-terminus, indicating a putatively secreted protein. No other domains were predicted, suggesting that the RsLysM has a modular structure similar to the already characterized homologs from filamentous ascomycetes. To test if this effector candidate is involved in virulence, we heterologously expressed it in the sugar beet hemibiotroph fungal pathogen $C$. beticola. Using RT-PCR and Western blot, we confirmed the expression of this candidate effector in RsLysM+ strains (Fig. S1). The RsLysM protein was also detected in RsLysM+ culture filtrates, confirming that it is a secreted one (Fig S1b). Phenotypic analysis on these strains displayed no difference in morphology, growth rate or conidiation in comparison to wild type (Ty1) (data not shown). The RsLys $M+C$. beticola strains displayed significantly larger necrotic lesions ( $\mathrm{p}$ value $=0.032$ ) on sugar beet leaves compared to wild type and strains where only the vector was inserted (Fig. 2a, b). The fungal biomass was significantly increased ( $p$ value $=0.003$ ) in comparison with wild-type and empty vector for RsLys $M+$ strains, further supporting the role of these candidate effectors in host colonization (Fig. 2c). This probably is attributed to the involvement of the RsLyM effector in an initial biotrophic phase of C. beticola.

\section{The RsLysM effector binds to chitin}

Previous analyses of LysM effector proteins from plant pathogenic ascomycetes have demonstrated their ability to bind chitin (de Jonge et al. 2010; Marshall et al. 2011; Kombrink et al. 2017). Whether LysM effectors from basidiomycetes have a similar chitin-binding affinity is unknown. Alignment of RsLysM domains with Ecp6 homologs from C. fulvum, showed a conserved motif, indicating that RsLysM could also be a chitin-binding protein (Fig. 3a). Therefore, we expressed the RsLysM in P. pastoris and purified the protein, which was used for the chitin-binding assay. The RsLysM protein was able to interact with all tested forms of chitin, such as chitin beads, crab shell chitin and chitosan (Fig. 3b). No precipitation with other polysaccharides such as xylan and cellulose was observed (Fig. 3b). Together, these data suggest that the RsLysM is an active chitin-binding effector protein, similar to other already characterized LysM effectors from filamentous ascomycetes.

\section{The RsLysM effector suppresses chitin-triggered immunity but does not protect hyphae from degradation}

It is known that LysM effectors assist filamentous ascomycetes avoiding plant immunity responses triggered by chitin (de Jonge et al. 2010; Marshall et al. 2011; Mentlak et al. 2012; Kombrink et al. 2017). To investigate whether the RsLysM effector also prevents plant chitin-triggered immunity, $N$. benthamiana leaves were treated with $10 \mu \mathrm{M}$ chitin oligomers (GlcNAc) $)_{6}$, which led to reactive oxygen species (ROS) burst (Fig. 3c). Addition of $10 \mu \mathrm{M}$ RsLysM pure protein led to a significant reduction of ROS (Fig. 3c), suggesting that RsLysM displays similar non-recognition function as seen in other pathosystems. Certain LysM effectors are able to protect fungal hyphae from chitinolytic activity (Marshall et al. 2011; Kombrink et al. 2017) similar to the Avr4 effector from C. fulvum (van den Burg et al. 2006). To 


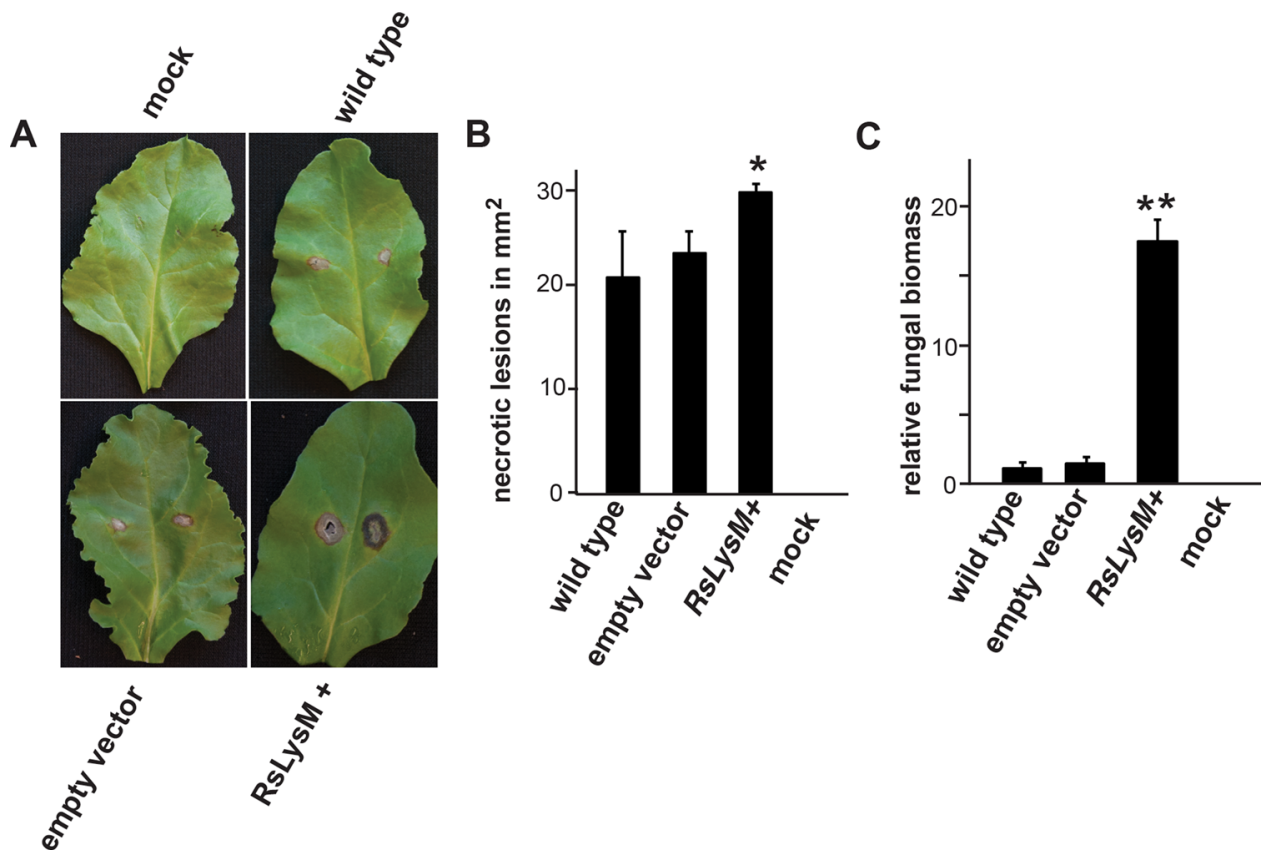

Fig. 2 Assays of the RsLySM effector. a Symptoms on sugar beet leaves in response to $C$. beticola strains harboring the RsLySM gene $(R s L y s M+)$ driven by the $g d p A$ promoter, $7 \mathrm{dpi}$. b Area of necrotic lesions on sugar beet leaves. c Fungal biomass quantification upon infection of sugar beet leaves. For quantitative PCR (qPCR), the $C$. beticola actin (act) gene was used. Data were normalized with the

investigate whether the RsLysM displays a similar function, germinated conidia from $T$. viride were mixed with pure RsLysM protein, while Avr4 and BSA were used as positive and negative controls, respectively. Our result showed that RsLysM was unable to protect fungal hyphae against degradation from bacterial chitinases and zymolyases, in contrast to Avr4 (Fig. 4).

\section{Discussion}

Rhizoctonia solani is a fungus commonly described as a saprophyte, thriving on dead or dying plant debris/cells that is able to switch to a pathogenic endotrophic growth. A complete understanding of the switch of lifestyles from endophytic to a parasitic form is still lacking. Necrotrophic pathogens secrete mainly effectors designed as host-specific or host-selective toxins able to promote necrosis (Vleeshouwers and Oliver 2014). In an R. solani AG8 strain, attacking both monocots and dicots, a xylanase and a protease inhibitor I9 induced cell death when expressed in Nicotiana benthamiana (Anderson et al. 2017). Similarly, cell death induction was observed in rice, maize and soybean leaves, when purified candidate effectors from an AG1-1A strain, elongation factor gene (elf-1) from Beta vulgaris. Data show the average of three independent strains and each set includes three biological replicates. Asterisks indicate statistical significant differences between the WT, empty vector and transgenic $C$. beticola strains according to Fisher's test $(* p$ value $<0.05, * * p$ value $<0.01)$. Error bars represent standard deviation

causing rice sheath blight, were evaluated (Zheng et al. 2013).

The LysM domain is ubiquitous and can be found across all kingdoms. The LysM module recognizes the GlcNAc$\mathrm{X}$-GlcNAc sequence in polysaccharides (Buist et al. 2008) and details about the binding characteristics have been clarified (Mesnage et al. 2014). Chitin is the main component of fungal cell walls (Bowman and Free 2006) and serves as a microbe-associated molecular pattern (MAMP), recognized by plant receptors triggering immune responses (Thomma et al. 2011). The most common function of LysM molecules is to perturb chitin-triggered immunity, but it may also have other roles. For instance, a LysM effector is required for the appressorium function in Colletotrichum higginsianum (Takahara et al. 2016). A number of LysM effectors have been identified in plant pathogenic ascomycete species mainly with hemibiotroph lifestyle, while limited studies have been conducted in mycoparasites and in mammalian fungal pathogens (de Jonge and Thomma 2009; Seidl-Seiboth et al. 2013). In the mycoparasitic species Trichoderma atroviride, several LysM genes that are clustered together with group $\mathrm{C}$ chitinases in the genome, were co-regulated with chitinase genes during interaction with $B$. cinerea (Gruber et al. 2011). Their role is unknown, but probably they serve similar function, upon attack by fungal prey (Tzelepis and Karlsson 2019). 
Fig. 3 Functional assays of the RsLysM protein. a Alignment between RsLysM and Ecp6 CBM50 domains. Sequence similarities are depicted with black shade. b Binding affinity assay on different insoluble polysaccharides. $20 \mu \mathrm{g} / \mathrm{ml}$ of LysM protein was mixed with $5 \mathrm{mg}$ of chitin beads, crab shell chitin, chitosan, xylan or cellulose. Presence of protein in bound (pellet) and unbound fraction (supernatant) separated using SDS-polyacrylamide gel electrophoresis followed by Coomassie staining. c Chitininduced oxidative (ROS) burst assay in $N$. benthamiana leafs. Production of ROS was determined using luminol-dependent chemiluminescence. Leaf disks were treated with $10 \mathrm{nM}$ flagellin (positive control), $10 \mu \mathrm{M}$ $(\mathrm{GlcNAc})_{6}, 10 \mu \mathrm{M}$ RsLySM protein, $10 \mu \mathrm{M}(\mathrm{GlcNAc})_{6}+10$ $\mu \mathrm{M}$ RsLySM protein. The luminol reagent was used as a negative control. At least eight biological replicates were run, and the experiment repeated twice
A
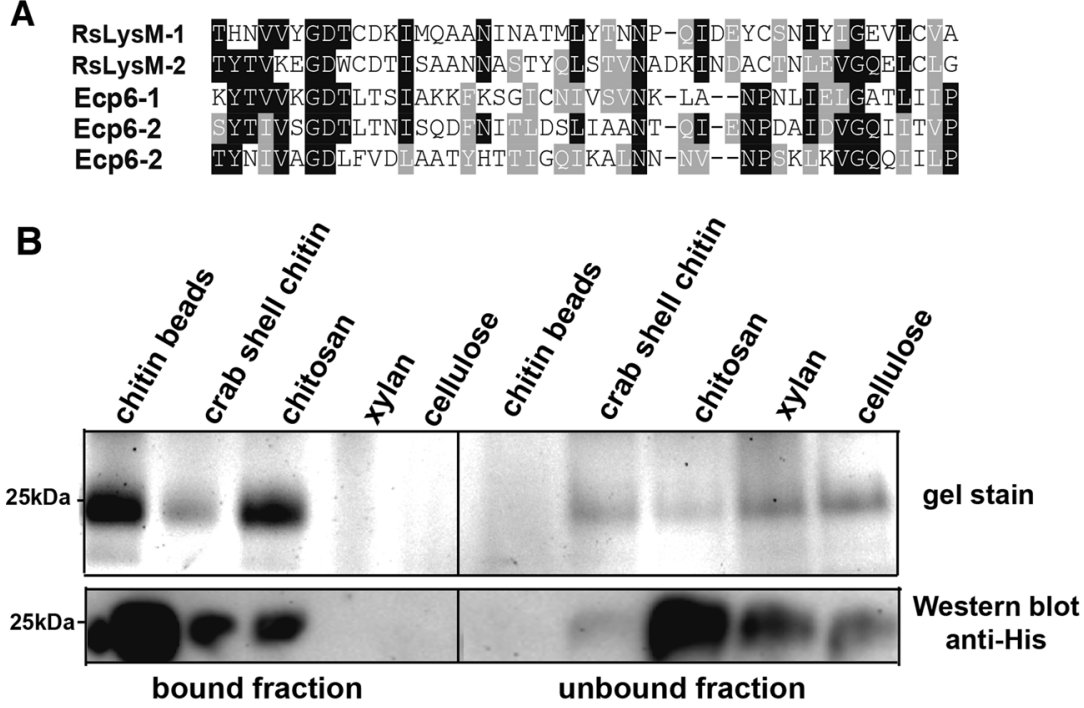

C

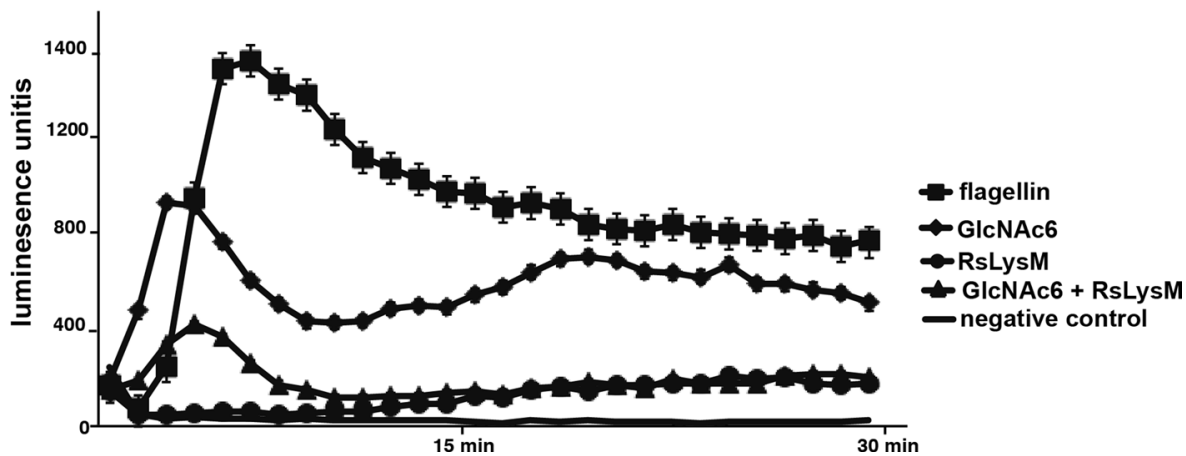

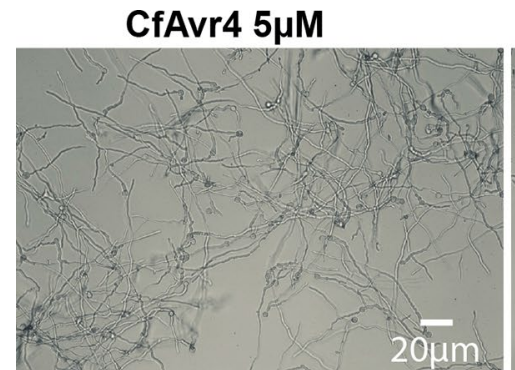

CfAvr4 5 $\mu \mathrm{M}$

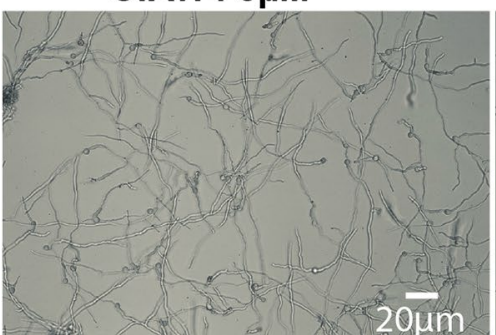

RsLysM $10 \mu M$

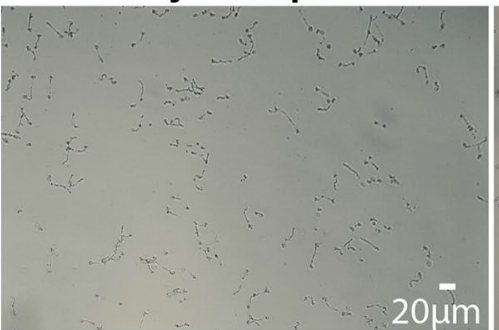

RsLysM 20 $\mu$ M

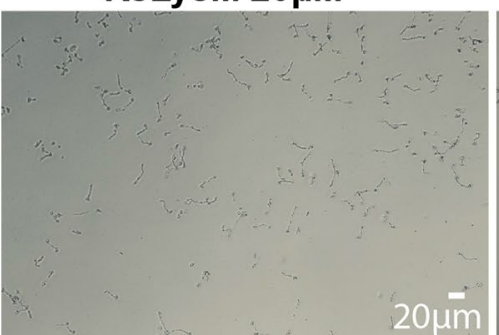

BSA $5 \mu \mathrm{M}$

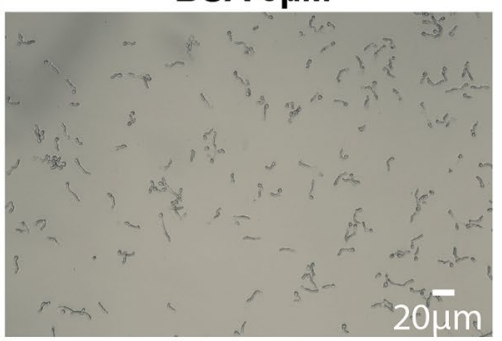

BSA $5 \mu M$

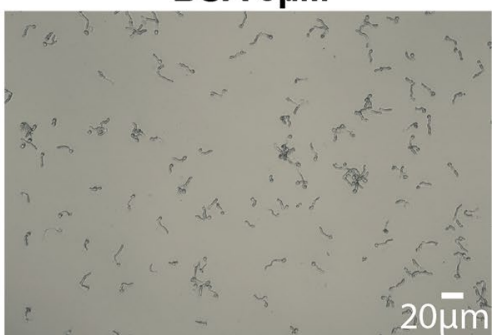

Fig. 4 Hyphal protection assay of the RsLysM effector. 10 and $20 \mu \mathrm{M}$ of the RsLysM pure protein was incubated with $3 \times 10^{5} \mathrm{~T}$. virens conidia for $30 \mathrm{~min}$ and $0.1 \mathrm{U}$ bacterial chitinase and $10 \mathrm{U}$ zymolyase.
Images were taken at 6 hpi. $5 \mu \mathrm{M}$ Avr4 from C. fulvum and bovine serum albumin (BSA) were used as positive and negative controls, respectively 
In this study, we investigated the role of a LysM candidate effector protein predicted as a singleton in the $R$. solani AG22IIIB strain (Wibberg et al. 2016b). This effector showed induction upon infection and suppressed ROS burst in a heterologous system of plant disease. This is in agreement with the previously published data from foliar pathogens such as $C$. fulvum and Zymoseptoria tritici (de Jonge et al. 2010; Marshall et al. 2011). However, the situation is different in another plant pathogen Verticillium dahliae, where three genes, encoded for core LysM effectors, were not induced upon infection (Kombrink et al. 2017). The $C$. beticola strains expressing RsLysM displayed increased fungal biomass and necrotic lesions in infected sugar beet plants, strongly supporting its role in host colonization. Similarly, the lineage-specific Vd2LysM and Ecp6 effectors are required for full virulence of $V$. dahliae and C. fulvum, respectively, since deletion of these genes led to reduced disease symptom development (Bolton et al. 2008; de Jonge et al. 2013). Heterologous expression of Ecp6 in Fusarium oxysporum led to increased virulence (Bolton et al. 2008), similar to RsLysM in C. beticola. In contrast, deletion of three core LysM effectors from the $V$. dahliae JR2 strain showed no changes in pathogen virulence (Kombrink et al. 2017), indicating no or minor role in disease development. RsLysM did not prevent fungal hyphae from degradation, which indicates that it is not involved in protecting the fungal cell wall from external hydrolytic enzymes, similar to the Epc6 LysM effector from C. fulvum (de Jonge et al. 2010). C. beticola genome (PRJNA270309) contains three genes (XP_023450473, XP_023449982 and XP_023450473.1) that encode putative LysM effectors. At present, no transcript or functional data are available on these genes. As we described above, not all LysM effectors contribute to virulence, thus it is possible that these three putative LysM proteins play a minor role in $C$. beticola virulence, in contrast to the RsLysM. By now, no LysM effectors have been characterized in any plant pathogenic species belonging to Basidiomycota, despite their presence in the sequenced genomes (Wibberg et al. 2013, 2016a; Zheng et al. 2013; Cubeta et al. 2014; Hane et al. 2014). As described above, LysM effectors can have multiple functions thus, additional roles of RsLysM in the lifecycle of $R$. solani such as in the penetration process, cannot be excluded.

In conclusion, our data show that $R$. solani might utilize a LysM effector to evade chitin-triggered immunity similar to hemibiotrophic pathogens. It seems that $R$. solani does not rely only on necrosis-inducing effectors to establish a successful infection. Whether an initial biotrophic stage is required for the $R$. solani infection process remains to be elucidated.

Acknowledgements The authors want to acknowledge: Prof. Bart Thomma, Peter Herfs, Nick Snelders and Hui Tian at Wageningen University, Phytopathology Lab, who contributed to RsLysM protein production and purification, MariboHilleshög Research that provided C. beticola Ty1 strain and sugar beet seeds (breeding line 16045118 01), Dr. Ioannis Stergiopoulos and Dr. Li Hung-Chen at UC Davis,
Phytopathology Lab for helping us with the hyphal protection assay. This work was supported by grants from: the Swedish Research Council VR (Grant number 621-2012-3982) MariboHilleshög Research and the Swedish University of Agricultural Sciences.

\section{Compliance with ethical standards}

Conflict of interest The authors declare that they have no conflict of interest.

Open Access This article is distributed under the terms of the Creative Commons Attribution 4.0 International License (http://creativeco mmons.org/licenses/by/4.0/), which permits unrestricted use, distribution, and reproduction in any medium, provided you give appropriate credit to the original author(s) and the source, provide a link to the Creative Commons license, and indicate if changes were made.

\section{References}

Adams GC (1988) Thanatephorus cucuemeris (Rhizoctonia solani) a species complex of wide host range. Adv Plant Pathol 6:535-592

Adams GC, Butler EE (1983) Influence of nutrition on the formation of basidia and basidiospores in Thanatephorus cucumeris. Phytopathology 73:147-151

Anderson NA (1982) The genetics and pathology of Rhizoctonia solani. Annu Rev Phytopathol 20:329-347

Anderson JP, Sperschneider J, Win J, Kidd B, Yoshida K, Hane J, Saunders DGO, Singh K (2017) Comparative secretome analysis of Rhizoctonia solani isolates with different host ranges reveals unique secretomes and cell death inducing effectors. Sci Rep 7:10410

Bernardes-de-Assis J, Storari M, Zala M, Wang W, Jiang D, Shidong L, Jin M, McDonald BA, Ceresini PC (2009) Genetic structure of populations of the rice-infecting pathogen Rhizoctonia solani AG-1 IA from China. Phytopathology 99:1090-1099

Bolton MD, van Esse HP, Vossen JH, de Jonge R et al (2008) The novel Cladosporium fulvum lysin motif effector Ecp6 is a virulence factor with orthologues in other fungal species. Mol Microbiol 69:119-136

Bolton MD, Panella L, Campbell L, Khan MF (2010) Temperature, moisture, and fungicide effects in managing Rhizoctonia root and crown rot of sugar beet. Phytopathology 100:689-697

Bowman SM, Free SJ (2006) The structure and synthesis of the fungal cell wall. BioEssays 28:799-808

Buddemeyer J, Pfähler B, Petersen J, Märländer B (2004) Genetic variation in susceptibility of maize to Rhizoctonia solani (AG22IIIB) - symptoms and damage under field conditions in Germany. J Plant Dis Prot 111:521-533

Buhre C, Kluth C, Bürcky K, Märländer B, Varrelmann M (2009) Integrated control of root and crown rot in sugar beet: combined effects of cultivar, crop rotation, and soil tillage. Plant Dis 93:155-161

Buist G, Steen A, Kok J, Kuipers OP (2008) LysM, a widely distributed protein motif for binding to (peptido)glycans. Mol Microbiol 68:838-847

Ceresini PC, Shew DH, Vilgalys RJ, Rosewich LU, Cubeta MA (2002) Genetic structure of populations of Rhizoctonia solani AG-3 on potato in eastern North Carolina. Mycologia 94:450-460

Chamoun R, Samsatly J, Pakala SB, Cubeta MA, Jabaji S (2015) Suppression subtractive hybridization and comparative expression of a poreforming toxin and glycosyl hydrolase genes in Rhizoctonia solani during potato sprout infection. Mol Genet Genom 290:877-900

Crespo-Sempere A, López-Pérez M, Martínez-Culebras PV, GonzálezCandelas L (2011) Development of a green fluorescent-tagged strain 
of Aspergillus carbonarius to monitor fungal colonization in grapes. Int J Food Microbiol 148:135-140

Cubeta MA, Thomas E, Dean RA, Jabaji S et al (2014) Draft genome sequence of the plant-pathogenic soil fungus Rhizoctonia solani anastomosis group 3 strain Rhs1AP. Genome Announc 2:e01072-14

de Jonge R, Thomma BPHJ (2009) Fungal LysM effectors: extinguishers of host immunity? Trends Microbiol 17:151-157

de Jonge R, van Esse HP, Kombrink A, Shinya T et al (2010) Conserved fungal LysM effector Ecp6 prevents chitin-triggered immunity in plants. Science 329:953-955

de Jonge R, Bolton MD, Kombrink A, van den Berg GC, Yadeta KA, Thomma BP (2013) Extensive chromosomal reshuffling drives evolution of virulence in an asexual pathogen. Genome Res 23:1271-1282

Gonzalez M, Pujol M, Metraux JP, Gonzalez-Garcia V, Bolton MD, Borrás-Hidalgo O (2011) Tobacco leaf spot and root rot caused by Rhizoctonia solani Kuhn. Mol Plant Pathol 12:209-216

Gruber S, Vaaje-Kolstad G, Matarese F, Lopez-Mondejar R, Kubicek CP, Seidl-Seiboth V (2011) Analysis of subgroup C of fungal chitinases containing chitin-binding and LysM modules in the mycoparasite Trichoderma atroviride. Glycobiology 21:122-133

Hane JK, Anderson JP, Williams AH, Sperschneider J, Singh KB (2014) Genome sequencing and comparative genomics of the broad hostrange pathogen Rhizoctonia solani AG8. PLoS Genet 10:e1004281

Hemetsberger C, Herrberger C, Zechmann B, Hillmer M, Doehlemann G (2012) The Ustilago maydis effector Pep1 suppresses plant immunity by inhibition of host peroxidase activity. PLoS Pathog 8:e1002684

Kohler AC, Chen LH, Hurlburt N, Salvucci A, Schwessinger B, Fisher AJ, Stergiopoulos I (2016) Structural analysis of an Avr4 effector ortholog offers insight into chitin binding and recognition by the Cf-4 receptor. Plant Cell 28:1945-1965

Kombrink A, Rovenich H, Shi-Kunne X, Rojas-Padilla E et al (2017) Verticillium dahliae LysM effectors differentially contribute to virulence on plant hosts. Mol Plant Pathol 18:596-608

Letunic I, Doerks T, Bork P (2009) SMART 6: recent updates and new developments. Nucleic Acids Res 37:D229-D232

Livak KJ, Schmittgen TD (2001) Analysis of relative gene expression data using real-time quantitative PCR and the 2(-delta delta $C(T)$ ) method. Methods 25:402-408

Lo Presti L, Lanver D, Schweizer G, Tanaka S, Liang L, Tollot M, Zuccaro A, Reissmann S, Kahmann R (2015) Fungal effectors and plant susceptibility. Annu Rev Plant Biol 66:513-545

Ma KW, Ma W (2016) Phytohormone pathways as targets of pathogens to facilitate infection. Plant Mol Biol 91:713-725

Marcuzzo LL, Haveroth R, Nascimento A (2015) Induction technique of sporulation in vitro of Cercospora beticola. Summa Phytopathol 41:74

Marshall R, Kombrink A, Motteram J, Loza-Reyes E, Lucas J, HammondKosack KE, Thomma BP, Rudd JJ (2011) Analysis of two in planta expressed LysM effector homologs from the fungus Mycosphaerella graminicola reveals novel functional properties and varying contributions to virulence on wheat. Plant Physiol 156:756-769

Mentlak TA, Kombrink A, Shinya T, Ryder LS et al (2012) Effectormediated suppression of chitin-triggered immunity by Magnaporthe oryzae is necessary for rice blast disease. Plant Cell 24:322-335

Mesnage S, Dellarole M, Baxter NJ, Rouget JB et al (2014) Molecular basis for bacterial peptidoglycan recognition by LysM domains. Nat Commun 5:4269

Möller EM, Bahnweg G, Sandermann H, Geiger HH (1992) A simple and efficient protocol for isolation of high molecular weight DNA from filamentous fungi, fruit bodies and infected plant tissues. Nucleic Acids Res 20:6115-6116

Nikou D, Malandrakis A, Konstantakaki M, Vontas J, Markoglou A, Ziogas B (2009) Molecular characterization and detection of overexpressed C-14 alpha-demethylase-based DMI resistance in Cercospora beticola field isolates. Pestic Biochem Physiol 95:18-27

Ogoshi A (1987) Ecology and pathogenicity of anastomosis and intraspecific groups of Rhizoctonia solani Kühn. Annu Rev Phytopathol 25:125-143

Petersen TN, Brunak S, von Heijne G, Nielsen H (2011) SignalP 4.0: discriminating signal peptides from transmembrane regions. Nat Methods 8:785-786

Preston GM (2007) Metropolitan microbes: type III secretion in multihost symbionts. Cell Host Microbe 2:291-294

Qutob D, Kemmerling B, Brunner F, Küfner I et al (2006) Phytotoxicity and innate immune responses induced by Nep1-like proteins. Plant Cell 18:3721-3744

Rooney HC, Van't Klooster JW, van der Hoorn RA, Joosten MH, Jones JD, de Wit PJ (2005) Cladosporium Avr2 inhibits tomato Rcr3 protease required for $\mathrm{Cf}$-2-dependent disease resistance. Science 308:1783-1786

Schulze S, Koch HJ, Märländer B, Varrelmann M (2016) Effect of sugar beet variety and non-host plant on Rhizoctonia solani AG2-2IIIB soil inoculum potential measured in soil DNA extracts. Phytopathology 106:1047-1054

Seidl-Seiboth V, Zach S, Frischmann A, Spadiut O et al (2013) Spore germination of Trichoderma atroviride is inhibited by its LysM protein TAL6. FEBS J 280:1226-1236

Stergiopoulos I, de Wit PJGM (2009) Fungal effector proteins. Annu Rev Phytopathol 47:233-263

Takahara H, Hacquard S, Kombrink A, Hughes HB et al (2016) Colletotrichum higginsianum extracellular LysM proteins play dual roles in appressorial function and suppression of chitin-triggered plant immunity. New Phytol 211:1323-1337

Thomma BPHJ, Nürnberge T, Joosten MHAJ (2011) Of PAMPs and effectors: the blurred PTI-ETI dichotomy. Plant Cell 23:4-15

Tzelepis G, Karlsson M (2019) Killer toxin-like chitinases in filamentous fungi: structure, regulation and potential roles in fungal biology. Fungal Biol Rev 33:123-132

Tzelepis G, Melin P, Jensen-Funck D, Stenlid J, Karlsson M (2012) Functional analysis of glycoside hydrolase family 18 and 20 genes in Neurospora crassa. Fungal Genet Biol 49:717-730

Utermark J, Karlovsky P (2008) Genetic transformation of filamentous fungi by Agrobacterium tumefaciens. Protocol Exchange. https://doi. org/10.1038/nprot.2008.83

van den Burg HA, Harrison SJ, Joosten MH, Vervoort J, de Wit PJ (2006) Cladosporium fulvum Avr4 protects fungal cell walls against hydrolysis by plant chitinases accumulating during infection. Mol Plant Microbe Interact 19:1420-1430

Vleeshouwers VGAA, Oliver RP (2014) Effectors as tools in disease resistance breeding against biotrophic, hemibiotrophic, and necrotrophic plant pathogens. Mol Plant Microbe Interact 27:196-206

Weiberg A, Wang M, Lin FM et al (2013) Fungal small RNAs suppress plant immunity by hijacking host RNA interference pathways. Science 342:118-123

Wibberg D, Jelonek L, Rupp O, Hennig M et al (2013) Establishment and interpretation of the genome sequence of the phytopathogenic fungus Rhizoctonia solani AG1-IB isolate 7/3/14. J Biotechnol 167:142-155

Wibberg D, Andersson L, Rupp O et al (2016a) Draft genome sequence of the sugar beet pathogen Rhizoctonia solani AG2-2IIIB strain BBA69670. J Biotechnol 222:11-12

Wibberg D, Andersson L, Tzelepis G et al (2016b) Genome analysis of the sugar beet pathogen Rhizoctonia solani AG2-2IIIB revealed high numbers in secreted proteins and cell wall degrading enzymes. BMC Genom 17:245

Zheng A, Lin R, Zhang D et al (2013) The evolution and pathogenic mechanisms of the rice sheath blight pathogen. Nat Commun 4:14

Publisher's Note Springer Nature remains neutral with regard to jurisdictional claims in published maps and institutional affiliations. 